\title{
NUTRI Quiz
}

1. The functionally active form ofvitamin-

$\mathrm{D}$ is

a)Cholecalciferol

b)Ergocalciferol

c) Dehydrocholesterol

d) 1,2,5Dihydroxycholecalciferol

2 Arrange the following foods in decreasing order of vitamin-B 12 content 1)Liver 2)fish 3)mutton4)milk
a) $1,2,3,4$
b) $2,3,4,1$
c) $3,1,2,4$
d) $4,2,1,3$

3. Colostrum is richin
a) Carbohydrate
b) Minerals
c) Vitamins
d) Antibodies

4. From which plant source gluten isderived
a) Soya
b) Rice
c) Corn
d) Wheat

5. HDL is synthesized \& secretedfrom
a) Pancreas
b) Liver
c) Kidney
d) Muscles

6. Germination enhance followingsnutrients

1)Vitamin-B 2) vitamin - C 3)Protein

4) Carbohydrate
a) Only2
b) $1 \& 2$
c) $3 \& 4$
d) all of these

7. Assertion (A) it is advised to restrict sodium in the diet of hypersensitive patients Reasoning $(\mathrm{R})$ sodium restricted diets are given in oliguria

a) Both a \& r are true \& $r$ is a correct expantion fora

b) Both a \& $r$ are true, but $r$ is not the correct expantion
c) Both a \& r arefalse
d) $\mathrm{A}$ is false, but $\mathrm{r}$ is a correct expantion of A

8. Daily Zinc requirement of for an adult is
a) $12 \mathrm{mg}$
b) $8 \mathrm{mg}$
c) $350 \mathrm{mg}$
d) $600 \mathrm{mg}$

9. Which of the following is not under anthropometrymethod
a) Skin FoldThickness
b) WaistCircumference
c) BloodPressure
d) MidUpperArm Circumference

10. Cutlet is prepared using whichmethod
a) Pouching
b) Braising
c) Boiling
d) Frying Answers will be discussed in next issue 